\title{
A Framework for End-to-End Verification and Evaluation of Register Allocators
}

\author{
V. Krishna Nandivada ${ }^{1}$, Fernando Magno Quintão Pereira ${ }^{2}$, \\ and Jens Palsberg ${ }^{2}$ \\ ${ }^{1}$ IBM India Research Laboratory, Delhi \\ ${ }^{2}$ UCLA Computer Science Department, University of California, Los Angeles
}

\begin{abstract}
This paper presents a framework for designing, verifying, and evaluating register allocation algorithms. The proposed framework has three main components. The first component is MIRA, a language for describing programs prior to register allocation. The second component is FORD, a language that describes the results produced by the register allocator. The third component is a type checker for the output of a register allocator which helps to find bugs. To illustrate the effectiveness of the framework, we present RALF, a tool that allows a register allocator to be integrated into the gcc compiler for the StrongARM architecture. RALF simplifies the development of register allocators by sheltering the programmer from the internal complexity of gcc. MIRA and FORD's features are sufficient to implement most of the register allocators currently in use and are independent of any particular register allocation algorithm or compiler. To demonstrate the generality of our framework, we have used RALF to evaluate eight different register allocators, including iterated register coalescing, linear scan, a chordal based allocator, and two integer linear programming approaches.
\end{abstract}

\section{Introduction}

\subsection{Background}

The register allocator is one of the most important parts of a compiler. Our experiments show that an optimal algorithm can improve the execution time of the compiled code by up to $250 \%$. Although researchers have studied register allocation for a long time, many interesting problems remain. For example, in recent years PLDI (ACM SIGPLAN Conference on Programming Language Design and Implementation) has published several papers on register allocation [2004 (2 papers), 2005 (3 papers), 2006 (2 papers)]. While the essence of register allocation is well understood, developing a high-quality register allocator is nontrivial. In addition to understanding the register allocation algorithm, which can be complex, the developer must also know the internals of the compiler where the allocator will be implemented. For example, public domain compilers such as GCC [2] or SMLNJ [1] and compiler frameworks such as SUIF 15] or SOOT 31] allow a programmer to implement a new register allocator. However, the programmer has to understand and work with their data structures, 
which are complicated because register allocation affects both the machine specific and machine independent parts of the compilation process. One attempt to address this problem was Tabatabai et al's. 3 register allocation framework, implemented in the CMU C compiler. Their framework presents modules (for example, graph construction, coalescing, color assignment, spill code insertion, and others) that different register allocators might need. However, if the allocator needs mechanisms other than those provided by the framework, the programmer must still deal with the internals of the CMU C compiler. A goal of our work is to completely shield the developer of register allocators from the internal complexities of a compiler.

Debugging register allocators is also a complicated task. Errors may surface in non-trivial ways; sometimes many instructions after the incorrect code. Moreover, the low-level nature of the machine code and its large size makes visual inspection of the register-allocator's output tedious and error-prone. As a testimony of these difficulties, most recent publications in this field report only static data and not run-time measurements, let alone implementations in industrial compilers. Although static data (such as number of spills, and number of registers used) is important, it does not reflect the behavior of the register allocator in the presence of other optimizations and run time factors.

A few researchers have developed techniques for proving register allocators correct. Naik and Palsberg [21] proved the correctness of the ILP-based register allocator of Appel and George 6. Ohori 26 designed a register allocation algorithm as a series of proof transformations which is correct by construction. Other researchers have shown how to validate the output of register allocators. Necula [24] presented a translation validation infrastructure for the gcc compiler that includes register allocation. Necula's scheme treats the memory address of a spilled register as a variable, which allows reasoning about its live ranges, although relying on specific characteristics of the gcc compiler, such as addressing modes. Leroy [17] formally describes a technique to validate the output of graph coloring based register allocation algorithms. Basically, if the interference graph contains a pair of adjacent temporaries allocated to the same register, the verifier emits an error, otherwise it assumes that the code generated is correct. Andersson 5 and Pereira et al. 27 adopted similar approaches. Huang et al. [16] presented a more general approach which matches the live ranges of values in the original program against the live ranges of machine locations in the register-allocated program. A goal of our work is to use a type system to validate the output of register allocators, and to prove the soundness of the type system itself.

Annotations in the register-allocated code can help validation algorithms. Morrisett et al. 20 used type annotations to help guarantee memory safety, Necula and Lee [25] used more general annotations such as memory bounds, and Agat 4 used type annotations to validate the output of a register allocator. A goal of our work is to validate the output of register allocators without extra annotations in the target code. 


\subsection{Our Results}

We present a framework for designing, verifying, and evaluating register allocators. Our framework shields the developer from the internal complexities of a compiler, uses a type system to validate the output of register allocators, and does not rely on code annotations.

MIRA and FORD. Our framework centers around MIRA (Mathematical Intermediate representation for Register Allocation), a language for describing programs prior to register allocation, and FORD (FOrmat for Register allocation Directives), a language that describes the results produced by the register allocator. MIRA sources are abstract intermediate representations of programs immediately before the register allocation phase. MIRA descriptions contain architecture and program specific information. The former includes information such as number and classes of machine registers, number of caller-save registers, and costs of loads and stores. The latter consists of information such as the program's control flow graph, use and definition sites of each variable, and estimated usage frequency of each instruction. In the context of our framework, the register allocator emits FORD directives that control spill code generation, register and variable mapping at different program points, and any additional code that needs to be inserted (For example, move instructions). MIRA and FORD can accommodate many of the traditional register allocation algorithms and are simple enough to be easily used by the developer of register allocators.

Type system. We use a type system to verify that the output of a register allocator is correct. Our type system was inspired by Morrisett et al.'s type system for assembly language [20] and can be used with intermediate representations other than MIRA and FORD. A type correct program is guaranteed to have properties such as: (1) pseudos whose live ranges overlap are assigned to different registers, (2) live ranges of the same pseudo always reach a join point assigned to the same register, and (3) a live register is not overwritten before it is used. We have found that typical errors in the implementation of a register allocator violate these properties. The type checker points out the locations of the register-allocated code where these properties fail. We have proved type soundness for our type system using the Twelf Meta-theorem prover 32 .

RALF. Our tool RALF (Register ALlocation Framework) allows a programmer to plug a new register allocator into gcc, without requiring the programmer to know any details of gcc's implementation. RALF is an extra layer added on top of gcc, acting as a glue between gcc and the plugged-in register allocator. The new register allocator takes a MIRA program as input and gives a collection of FORD directives as output. The main objective of RALF is to be simple: when writing a register allocator compatible with our framework, the developer only has to write a program that translates MIRA to FORD. RALF treats the register allocator, which can be implemented in any language, as a black box whose only purpose is to translate MIRA sources (provided by RALF) to FORD directives (which are fed back to RALF). Given an input program and the plugged-in register allocator, RALF generates a StrongARM binary executable using the new 
register allocator and the rest of gcc. RALF uses our type checker to verify the output of the register allocator. In addition to our own experiments with RALF, we have used RALF in an advanced compiler course at UCLA. As part of a class assignment, each student implemented a different register allocation algorithm to be used with the framework. More about these experiences can be found at RALF's homepage http://compilers.cs.ucla.edu/ralf. Our current implementation of RALF compiles only C code to the StrongARM architecture; however, the MIRA and FORD description languages are designed to be general enough to fit other source languages and architectures. The RISC nature of the StrongARM architecture helps to keep RALF simple. Our implementation, which gets activated by different compiler switches, contains around 5000 lines of $\mathrm{C}$ code divided among 125 functions. The proposed framework is not intended for industrial implementations of register allocators, but for fast implementation and testing of research prototypes. The techniques used in our type system can be used also in a production compiler.

Comparison of eight register allocators. We have used RALF to implement and compare eight different registers allocators. The allocators tested range from classical algorithms, such as the usage-count based implementation [11, to novel approaches, such as register allocation via coloring of chordal graphs 27. In addition of using static data, such as number of variables spilled, we compare the different algorithms by running the produced code on a StrongARM processor.

The remainder of this paper is organized as follows: Section 2 describes a simplified version of MIRA and FORD and characterizes the register allocation problem. Section 3 presents our type system, and Section 4 discusses our experimental results.

\section{A Simplified View of MIRA and FORD}

Register allocation is the process of mapping a program $\mathcal{M}$ that can use an unbounded number of variables, or pseudo-registers, to a program $\mathcal{F}$ that must use a fixed (and generally small) number of machine registers to store data. In the remainder of this paper we use register in place of machine register, and pseudo for pseudo-register. If the number of registers is not sufficient to accommodate all the pseudos, some of them must be stored in memory; these are called spilled pseudos. Following the nomenclature normally used in the gcc community, we call the intermediate representation of programs $\mathcal{M}$ the Register Transfer Language (RTL) and we use Location Transfer Language ( $L T L$ ) to describe programs $\mathcal{F}$. As shown in Figure 1, MIRA and FORD have been designed to constitute an interface between the register allocator and the RTL/LTL intermediate representations. They facilitate the development of register allocation algorithms by hiding from the algorithm's designer the details of the RTL/LTL representation that are not relevant to the register allocation process. In order to precisely characterize the register allocation problem, we will use a simplified version of MIRA and a simplified version of FORD in this section. We will call them sMIRA and $\mathrm{sFORD}$ respectively. For our purposes, a register allocator $R A$ is a black box 


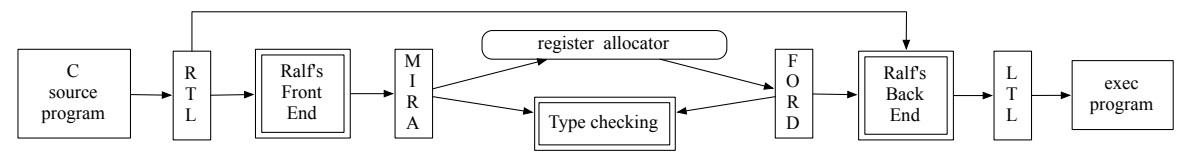

Fig. 1. Block diagram of our register allocation framework

which takes an sMIRA program $P_{s m}$ and a description of the target architecture, and produces an sFORD program $P_{s f}$. In this section, we will assume that the architecture specific information is a list of $K$ machine registers Regs, and a set of caller save registers CallerSave $\subseteq$ Regs. Thus, $P_{s f}=R A\left(P_{s m}\right.$, Regs, CallerSave). An actual register allocator would require more information, such as the class of each machine register, the cost of different operations, etc. Such information is present in the concrete specification of MIRA and FORD, which is given in the full version of this paper, available at http://compiler.cs.ucla.edu/ralf.

\subsection{Simplified MIRA}

sMIRA programs are described by the grammar in Figure 2(a). We adopt an abstract representation of programs. All the operands not relevant to register allocation, such as constants, heap memory addresses, and others, are represented with the symbol $\bullet$. The only explicit operands are pseudos $(p)$ and pre-colored registers $(r, p)$. The latter are pseudos that have been assigned a fixed machine register due to architectural constraints. In this simplified presentation, we only use pre-colored register to pass parameters to function calls, and to retrieve their return value. Other operands, such as constants and memory references on the heap are abstracted out.

An sMIRA program is a sequence of instruction blocks. Each instruction block consists of an address label, represented by $L$, heading a sequence of instructions (I) followed by a jump. sMIRA programs can use an unbounded number of pseudo registers $p$. We do not distinguish the opcode of instructions, except branches and function calls. Branches affect the control flow, and function calls may cause caller save registers to be overwritten, once register allocation has been performed. A function call such as $\left(r_{0}, p_{0}\right)=$ call $\left(r_{1}, p_{1}\right) . .\left(r_{s}, p_{s}\right)$ uses precolored pseudos $\left(r_{1}, p_{1}\right) . .\left(r_{s}, p_{s}\right)$ as parameters, and produces a return value in the pre-colored pseudo $\left(r_{0}, p_{0}\right)$. Notice that in sMIRA a call instruction does not contain a label; that is because we support only intra-procedural register allocation. An example of sMIRA program is given in Figure 2(b).

\subsection{Simplified FORD}

A register allocator produces SFORD programs, which are represented by the grammar in Figure 3(a). Operands in sFORD are bindings of pseudos $(p)$ to machine locations. In our representation, a machine location can be either a 


(Programs)
(Code labels)
(Instr. Sequence)
- (Jump)
- (Sequence)
(Pseudos)
(Registers)
(Operands)
- (Constants)
- (Pseudos)
- (Pre-coloreds)
(Instructions)
- (Assignment)
- (Ass. pre-col.)
- (Cond. jump)
- (Function call)

\section{(a)}

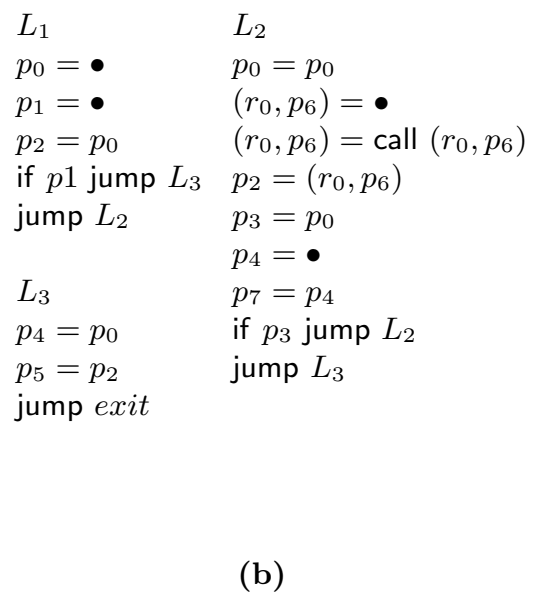

(b)

Fig. 2. (a) Syntax of sMIRA programs (b) Example of sMIRA program

\begin{tabular}{|c|c|c|c|}
\hline $\begin{array}{l}\text { (Programs) } \\
\text { (Code Labels) } \\
\text { (Inst. seq.) } \\
\text { - (Jump) } \\
\text { - (Sequence) } \\
\text { (Pseudos) } \\
\text { (Registers) } \\
\text { (Mem. locs.) } \\
\text { (Operands) } \\
\text { - (Constant) } \\
\text { - (Reg. bind) } \\
\text { - (Mem. bind) } \\
\text { (Instructions) } \\
\text { - (Assig.) } \\
\text { - (Store) } \\
\text { - (Cond. jump) } \\
\text { - (Func. call) }\end{array}$ & $\begin{aligned} P_{s f} & ::=L_{1} I_{1} ; \ldots ; L_{k} I_{k} \\
L & ::=L_{1}\left|L_{2}\right| \ldots \\
I & := \\
& \mid \text { jump } l \\
& \mid i ; I \\
p & ::=p_{1}\left|p_{2}\right| \ldots \\
r::= & \left|r_{1}\right| r_{2} \mid \ldots \\
l & :=l_{1}\left|l_{2}\right| \ldots \\
o::= & \\
\mid & \bullet \\
\mid & (r, p) \\
\mid & (l, p) \\
i::= & \\
\mid & (r, p)=o \\
& (l, p)=o \\
\mid & \text { if }(r, p) \text { jump } L \\
\mid & \left(r_{0}, p_{0}\right)=\text { call } \\
& \left(r_{1}, p_{1}\right) . .\left(r_{s}, p_{s}\right)\end{aligned}$ & $\begin{array}{l}L_{1} \\
\left(r_{1}, p_{0}\right)=\bullet \\
\left(r_{0}, p_{1}\right)=\bullet \\
\left(r_{1}, p_{2}\right)=\left(r_{1}, p_{0}\right) \\
\left(l_{0}, p_{2}\right)=\left(r_{1}, p_{2}\right) \\
\text { if }\left(r_{1}, p 1\right) \text { jump } L_{3} \\
\text { jump } L_{2} \\
L_{3} \\
\left(r_{1}, p_{4}\right)=\left(r_{1}, p_{0}\right) \\
\left(r_{0}, p_{2}\right)=\left(l_{0}, p_{2}\right) \\
\left(r_{0}, p_{5}\right)=\left(r_{0}, p_{2}\right) \\
\text { jump exit }\end{array}$ & $\begin{array}{l}L_{2} \\
\left(r_{1}, p_{0}\right)=\left(r_{1}, p_{0}\right) \\
\left(r_{0}, p_{6}\right)=\bullet \\
\left(l_{1}, p_{0}\right)=\left(r_{1}, p_{0}\right) \\
\left(r_{0}, p_{6}\right)=\operatorname{call}\left(r_{0}, p_{6}\right) \\
\left(r_{1}, p_{0}\right)=\left(l_{1}, p_{0}\right) \\
\left(r_{2}, p_{2}\right)=\left(r_{0}, p_{6}\right) \\
\left(l_{0}, p_{2}\right)=\left(r_{2}, p_{2}\right) \\
\left(r_{2}, p_{3}\right)=\left(r_{1}, p_{0}\right) \\
\left(r_{0}, p_{4}\right)=\bullet \\
\left(r_{0}, p_{7}\right)=\left(r_{0}, p_{4}\right) \\
\text { if }\left(r_{1}, p_{3}\right) \text { jump } L_{2} \\
\text { jump } L_{3}\end{array}$ \\
\hline & (a) & & (b) \\
\hline
\end{tabular}

Fig. 3. (a) Syntax of sFORD programs (b) Example of sFORD program 
physical register $(r)$, or a memory address $(l)$. In addition to calls and branches, we distinguish loads " $=(l, p)$ ", and stores " $(l, p)=$ ", because these instructions are used to save and restore spilled values. Notice that we make an explicit distinction between code labels, represented by $L$, and data labels (stack locations), represented by $l$. In the sFORD representation, caller-save registers can be overwritten by function calls; thus, the register allocator must guarantee that pseudos that are alive across function calls are not mapped to caller-save registers.

Let $P_{s m}$ be the sMIRA program in Figure 2(b), and consider an architecture where Regs $=\left\{r_{0}, r_{1}, r_{2}\right\}$ and CallerSave $=\left\{r_{0}, r_{1}\right\}$. Let $R A$ be a hypothetical register allocator, such that $P_{s f}=R A\left(P_{s m}\right.$, Regs, CallerSave $)$ is the program in Figure 3(b). In our example, $R A$ has allocated register $r_{1}$ to pseudo $p_{0}$ in the first instruction of $L_{1}$. The pseudo $p_{2}$ has been spilled due to the high register pressure in block $L_{2}$; its memory location is given by the label $l_{0}$. Furthermore, pseudo $p_{0}$ has been spilled to memory location $l_{1}$ because it is stored in the caller save register $r_{0}$ and is alive across a function call.

\section{$3 \quad$ Type Checking}

Inaccuracies in the implementation of a register allocation algorithm may result in different types of errors in the sFORD program. In Figure 4 we illustrate five different errors that can be produced by a flawed register allocator. In Fig. 4(a), $p_{0}$ was defined in register $r_{0}$ at instruction 1 , but it is expected to be found in register $r_{1}$ when used in instruction 2. In Fig. 4(b) register $r_{0}$ is overwritten in instruction 2 while it contains the live pseudo $p_{0}$. Fig. 4(c) describes a similar situation, but in this case a memory location is overwritten while the value it holds is still alive. In Fig. 4(d), we assume that $r_{1}$ is a caller save register. In this case, pseudo $p_{1}$ may have its location overwritten during the execution of the function call in instruction 2. Finally, in Fig. 4(e) the value of $p_{0}$, stored in register $r_{0}$ may be overwritten, depending on the path taken during the execution of the program. This last error is particularly elusive, because its consequences might not surface during the testing of the target program.

In order to guarantee that the values used in the sMIRA program are preserved in the sFORD representation, we use a type checker inspired by [20]. The basic data used in our type system are machine locations, and the type of a machine location is the pseudo-register that it stores. In our case, the register allocator

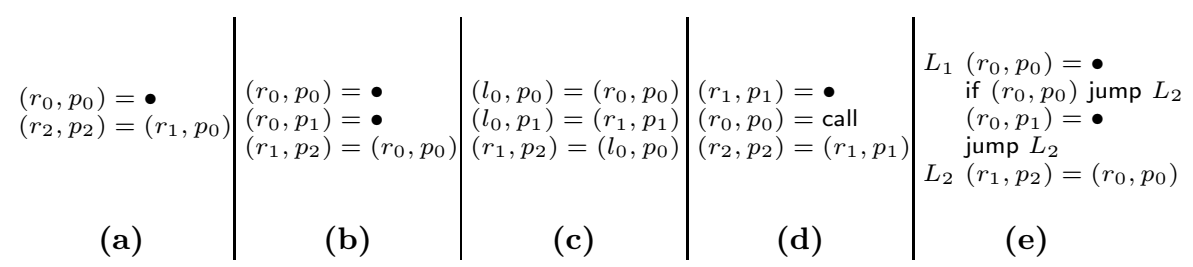

Fig. 4. (a-e) Examples of errors due to wrong register allocation 
annotates each definition or use of data with its type. A definition of a machine location, e.g $\left(r_{i}, p_{j}\right)=\bullet$ corresponds to declaring $r_{i}$ with the type $p_{j}$. Let $\left(r_{i}, p_{j}\right)$ be an annotated machine location. Intuitively, every time this machine location is used, e.g $(r, p)=\left(r_{i}, p_{j}\right)$, its annotated type corresponds to the type that can be discovered by a type inference engine if the sFORD program is correct. For instance, the program in Figure 4(a) is incorrect because $p_{0}$, the type of $r_{1}$ in the second instruction cannot be inferred. Notice that these annotations can be inferred from the sMIRA/sFORD programs; they are not present in the final LTL code.

\subsection{Operational Semantics of sFORD Programs}

We define an abstract machine to evaluate sFORD programs. The state $M$ of this machine is defined in terms of a tuple with four elements: $(C, D, R, I)$. If $M$ is a program state and we have $M^{\prime}$ such that $M \rightarrow M^{\prime}$, then we say that $M$ can take a step. A program state $M$ is stuck if $M$ cannot take a step. A program state $M$ goes wrong if $\exists M^{\prime}: M \rightarrow^{*} M^{\prime}$ and $M^{\prime}$ is stuck. $I$ is defined in Figure 3(a); the code heap $C$, data heap $D$, and register bank $R$ are defined below.

$$
\begin{array}{ll}
\text { (Code Heap) } & C::=\left\{L_{1}=I_{1}, \ldots, L_{k}=I_{k}\right\} \\
\text { (Data Heap) } & D::=\left\{l_{1}=p_{1}, \ldots, l_{m}=p_{m}\right\} \\
\text { (Register Bank) } & R::=\left\{r_{1}=p_{1}, \ldots, r_{n}=p_{n}\right\} \\
\text { (Machine State) } & M::=(C, D, R, I)
\end{array}
$$

The evaluation rules for our abstract machine are given in Figure 5. Rules 1 , 2 and 3 evaluate the operands of sFORD. The assignment statement (Rule 4) modifies the mapping in the register bank, and the store statement (Rule 5) modifies the mapping in the data heap. The result of a conditional branch has no importance in our representation. Therefore, an instruction such as if $(r, p)$ jump $v$ is evaluated non-deterministically by either Rule 6 or Rule 7 We conservatively assume that a call instruction changes the contents of all the caller save registers. It also defines a register with the return value (Rule 8). In order to simulate the effects of a function call on the caller-save registers we define the erasing function " $\diamond$ " below. We augment the set of pseudo-registers with $\perp$. This pseudo will be used as the type of non-initialized registers and we assume that it is not defined in any instruction of the original sMIRA program.

$$
\begin{aligned}
& \diamond: R \times X \mapsto R^{\prime} \\
& (R \diamond X)(r)=\perp \text { if } r \in X, \text { else } R(r)
\end{aligned}
$$

In Figure 5 the set $X$ is replaced by the set of caller-save registers. We draw the attention of the reader to the premises of rules 4 to 8 , which ensure that a location used by an instruction indeed contains the pseudo that is expected by that instruction. For example, for the sFORD instruction $\left(r_{1}, p_{1}\right)=\left(r_{0}, p_{0}\right)$, the premise of Rule 4 ensures that $r_{0}$ is holding the value $p_{0}$ when this instruction is executed. 


$$
\begin{aligned}
& D, R \vdash \bullet \\
& D, R \vdash(r, p) \text {, if } R(r)=p \wedge p \neq \perp \\
& D, R \vdash(l, p) \text {, if } D(l)=p \wedge p \neq \perp \\
& \frac{D, R \vdash o}{(C, D, R,(r, p)=o ; I) \rightarrow(C, D, R[r \mapsto p], I)} \\
& \frac{D, R \vdash o}{(C, D, R,(l, p)=o ; I) \rightarrow(C, D[l \mapsto p], R, I)} \\
& \frac{D, R \vdash(r, p)}{(C, D, R, \text { if }(r, p) \text { jump } L ; I) \rightarrow\left(C, D, R, I^{\prime}\right)} C_{c o n d} \\
& C_{\text {cond }}=L \in \operatorname{domain}(C) \wedge C(L)=I^{\prime} \\
& \frac{D, R \vdash(r, p)}{(C, D, R, \text { if }(r, p) \text { jump } L ; I) \rightarrow(C, D, R, I)} \\
& \frac{\forall\left(r_{i}, p_{i}\right), 1 \leq i \leq s, D, R \vdash\left(r_{i}, p_{i}\right)}{\left(C, D, R,\left(r_{0}, p_{0}\right)=\text { call }\left(r_{1}, p_{1}\right), \ldots,\left(r_{s}, p_{s}\right) ; I\right)} \\
& \rightarrow\left(C, D,(R \diamond \text { callerSave })\left[r_{0} \mapsto p_{0}\right], I\right) \\
& (C, D, R, \text { jump } L) \rightarrow(C, D, R, I) \text { if } C_{\text {jump }} \\
& C_{\text {jump }}=L \in \operatorname{domain}(C) \wedge C(L)=I
\end{aligned}
$$

Fig. 5. Operational Semantics of sFORD programs

\section{$3.2 \quad$ Typing Rules}

We define the following types for values:

$$
\text { value types } t::=p \mid \text { Const }
$$

We define three typing environments:

$$
\begin{aligned}
& \text { (Code heap type) } \Psi::=\left\{L_{1}:\left(\Gamma_{1} \times \Delta_{1}\right)\right. \\
&\left.\ldots, L_{k}:\left(\Gamma_{k} \times \Delta_{k}\right)\right\} \\
& \text { (Register bank type) } \Gamma::=\left\{r_{1}: p_{1}, \ldots, r_{m}: p_{m}\right\} \\
& \text { (Data heap type) } \Delta::=\left\{l_{1}: p_{1}, \ldots, l_{n}: p_{n}\right\}
\end{aligned}
$$

Operands that have no effect on the register allocation process are given the type Const. The type of a machine location (register or memory address) is determined 
Operands

$$
\begin{gathered}
\vdash \bullet: \text { Const } \\
\frac{\Gamma(r)=p \quad p \neq \perp}{\Gamma \vdash(r, p): p} \\
\frac{\Delta(l)=p \quad p \neq \perp}{\Delta \vdash(l, p): p}
\end{gathered}
$$

Instructions

$$
\begin{gathered}
\frac{\Delta ; \Gamma \vdash o: t \quad p \neq \perp}{\Psi \vdash(r, p)=o:(\Gamma \times \Delta) \mapsto(\Gamma[r: p] \times \Delta)} \\
\frac{\Delta ; \Gamma \vdash o: t \quad p \neq \perp}{\Psi \vdash(l, p)=o:(\Gamma \times \Delta) \mapsto(\Gamma \times \Delta[l: p])} \\
\frac{\Gamma \vdash(r, p): p \quad \Psi \vdash L:\left(\Gamma^{\prime} \times \Delta^{\prime}\right) \quad(\Gamma \times \Delta) \leq\left(\Gamma^{\prime} \times \Delta^{\prime}\right)}{\Psi \vdash \text { if }(r, p) \text { jump } L:(\Gamma \times \Delta) \mapsto(\Gamma \times \Delta)} \\
\frac{\forall\left(r_{i}, p_{i}\right), 1 \leq i \leq s, \Gamma \vdash\left(r_{i}, p_{i}\right): p_{i} \quad p_{0} \neq \perp}{\Psi \vdash\left(r_{0}, p_{0}\right)=\text { call }\left(r_{1}, p_{1}\right), \ldots,\left(r_{s}, p_{s}\right):(\Gamma \times \Delta) \mapsto\left((\Gamma \diamond \text { callerSave })\left[r_{0}: p_{0}\right] \times \Delta\right)}
\end{gathered}
$$

Instruction sequences

$$
\begin{gathered}
\frac{\Psi \vdash L:\left(\Gamma^{\prime} \times \Delta^{\prime}\right) \quad(\Gamma \times \Delta) \leq\left(\Gamma^{\prime} \times \Delta^{\prime}\right)}{\Psi \vdash \text { jump } L:(\Gamma \times \Delta)} \\
\frac{\Psi \vdash i:(\Gamma \times \Delta) \mapsto\left(\Gamma^{\prime} \times \Delta^{\prime}\right) \quad \Psi \vdash I:\left(\Gamma^{\prime} \times \Delta^{\prime}\right)}{\Psi \vdash i ; I:(\Gamma \times \Delta)}
\end{gathered}
$$

Bank of Registers

$$
\frac{\forall r \in \operatorname{domain}(\Gamma) . \vdash R(r): \Gamma(r)}{\Psi \vdash R \cdot \Gamma}
$$

Data Heap

$$
\frac{\forall l \in \operatorname{domain}(\Delta) . \vdash D(l): \Delta(l)}{\Psi \vdash D: \Delta}
$$

Code Heap

$$
\frac{\forall L \in \text { domain }(\Psi) \cdot \Psi \vdash C(L): \Psi(L)}{\vdash C: \Psi}
$$

Machine states:

$$
\frac{\vdash C: \Psi \quad \vdash D: \Delta \quad \vdash R: \Gamma \quad \Psi \vdash I:\left(\Gamma^{\prime} \times \Delta^{\prime}\right) \quad(\Gamma \times \Delta) \leq\left(\Gamma^{\prime} \times \Delta^{\prime}\right)}{\vdash(C, D, R, I)}
$$

Fig. 6. Type System of sFORD 
by the pseudo that is stored in that location. The environment $\Gamma$ contains the types of the machine registers, and the typing environment $\Delta$ contains the types of locations in the data heap. We will refer to $\Gamma$ and $\Delta$ as location environments. The environment $\Psi$ determines the type of each instruction block. The type of an instruction sequence is given by the minimum configuration of the bank of registers and data heap that the sequence must receive in order to be able to execute properly. We consider instructions as functions that modify the location environments, that is, an instruction $i$ expects an environment $(\Gamma \times \Delta)$ and returns a possibly modified environment $\left(\Gamma^{\prime} \times \Delta^{\prime}\right)$. We define an ordering on location environments as follows:

$$
\begin{gathered}
\Gamma \leq \Gamma^{\prime} \text { if } \forall r, r: p \in \Gamma^{\prime} \text { then } r: p \in \Gamma \\
\Delta \leq \Delta^{\prime} \text { if } \forall l, l: p \in \Delta^{\prime} \text { then } l: p \in \Delta \\
(\Gamma \times \Delta) \leq\left(\Gamma^{\prime} \times \Delta^{\prime}\right) \text { if } \Gamma \leq \Gamma^{\prime} \wedge \Delta \leq \Delta^{\prime}
\end{gathered}
$$

The type rules for sFORD programs are given in Fig. 6, According to rule 11, the type of a register binding such as $(r, p)$ is the temporary $p$, but, only if $p$ is the type of $r$ in the $\Gamma$ environment, otherwise it does not type-check. Similarly, Rule 12 determines the type of memory bindings. Rules 13,14 and 16 change the location environment. The ordering comparison in the premises of Rules 15 and 17 is necessary to guarantee that all the registers alive at the beginning of an instruction block have well defined types.

None of the programs in Fig. 4 type-check. In Fig. 4(a), $r_{1}$ is not declared with type $p_{0}$, thus the premise of Rule 11 is not satisfied. In Fig. 4(b), the type of $r_{0}$, before the execution of instruction 3 , is $p_{1}$, not $p_{0}$, as expected by the type annotation. Again, Rule 11 is not satisfied. Fig. 4(c) presents a similar case, but using a memory location instead of a register: the type of $l_{0}$ in instruction 3 is not $p_{0}$, as expected, but $p_{1}$. The type of the used operand would not satisfy the premise in Rule 12. In Fig. 4(d), $r_{1}$ has type $\perp$ before the execution of instruction 3 , which is different from the expected type $p_{1}$. Finally, in Fig. 过 $(\mathrm{e}) \Psi\left(L_{2}\right)=\left(\left[r_{0}: p_{0}\right] \times \Delta\right)$, but the type of instruction 4 is $\left(\left[r_{0}: p_{1}\right] \times \Delta\right) \mapsto\left(\left[r_{0}: p_{1}\right] \times \Delta\right)$. This would not satisfy the inequality in Rule 17 .

\subsection{Type Soundness}

We state the lemmas and theorems that constitute our soundness proof. Our soundness proof assumes that the sMIRA program defines each pseudo $p$ before $p$ is used. If the sFORD program type-checks, then it preserves the values alive in the original sMIRA code.

Theorem 1 (Preservation). If $\vdash M$, and $M \rightarrow M^{\prime}$, then $\vdash M^{\prime}$. 
Lemma 1 (Canonical Values). If $\vdash C: \Psi, \vdash D: \Delta$ and $\vdash R: \Gamma$ then:

1. If $\Psi \vdash L:(\Gamma \times \Delta)$, then $L \in$ domain $(C), C(L)=I$ and $\Psi \vdash I:(\Gamma \times \Delta)$.

2. If $\Delta ; \Gamma \vdash o: p$, then $o=r$, or $o=l$. If $o=r$, then $r \in \operatorname{domain}(R)$, else if $o=l$, then $l \in \operatorname{domain}(D)$.

3. If $\Delta ; \Gamma \vdash o$ : Const, then $o=\bullet$.

Theorem 2 (Progress). If $\vdash M$, then $M$ is a final state, or there exists $M^{\prime}$ such that $M \mapsto M^{\prime}$.

Corollary 1 (Soundness). If $\vdash M$, then $M$ cannot go wrong.

We have checked the proof using Twelf [32, and this proof can be found at http://compilers.cs.ucla.edu/ralf/twelf/

\subsection{Preservation of Callee-Save Registers}

Our type system is intra-procedural, and it assumes that a function call preserves callee-save registers. This must be verified for each procedure, after its typechecking phase, when every instruction has a well know type. If we assume that a machine register is either caller-save or callee-save, this verification step can be done via a simple test. Let $L_{0}$ be the label of the first instruction in the procedure, and let $L_{e}$ be an exit point. Let $\Psi\left(L_{0}\right)=\left(\Gamma_{0} \times \Delta_{0}\right)$, and let $\Psi\left(L_{e}\right)=\left(\Gamma_{e} \times \Delta_{e}\right)$. Callee-save registers are preserved at exit point $L_{e}$, if $\Gamma_{e} \diamond$ CallerSave $\leq \Gamma_{0} \diamond$ CallerSave.

Along with the preservation of callee-save registers, our type systems has a set of consistency requirements, which can be carried out as a sequence of table lookup verifications. These checks are explained in the full version of this paper, available at http://compiler.cs.ucla.edu/ralf.

\section{Experimental Results}

Figure 1 presents a high-level block diagram of RALF. RALF interfaces the transformation between the RTL and LTL intermediate representations used by gcc. RALF's front end consists mainly of gcc's parser, gcc's optimization phases, and code to produce a MIRA program from a RTL program. The back end consists mostly of a type checker, code for producing LTL instructions, and gcc's code generation engine. RALF interacts with the implementation of a register allocator via ordinary ASCII files. Given a RTL program $P$, RALF translates $P$ into a MIRA ASCII program $\mathcal{M}$, which is then fed to the plugged-in register allocator. The register allocator outputs a set of FORD directives $\mathcal{F}$, which are then given back to RALF. RALF checks that $(\mathcal{M}, \mathcal{F})$ is a correct mapping via the type system described in Section 3 applies the directives $\mathcal{F}$ on the original RTL program, and generates gcc's LTL code. RALF's back end does not need the original MIRA file in order to produce the LTL program. When producing the RTL code, RALF inserts loads and stores for callee save registers at the entrance and exit of each function (a smart register allocator might decide to take on that responsibility itself and RALF has an option for that). 
We have tested RALF with eight different register allocators: (1: gcc -O2) the allocator present in the gcc compiler, which has two main phases: (a) aggressive register allocation for local variables within basic blocks, (b) conservative allocation for the whole function. (2: Naive) The naive register allocator, which spills all the pseudos. (3: UBC) usage count based register allocator [11, (4: IRC) iterated register coalescing [13, (5: Chordal) register allocation via coloring of chordal graphs [27, (6: LS) linear scan [28, (7: RA) integer linear program (ILP) 23, (8: SARA) stack location allocation combined with register allocation (SARA) 23.

Five of the register allocators have been implemented in Java $(2,3,4,5$ and 6$)$. Algorithms 7 and 8 have been implemented in AMPL [10. The interface provided by RALF is extremely simple, and most of the code used to parse and output MIRA/FORD files could be reused among the different implementations. Table 7 compares the size of each implementation; (J) stands for Java, and (A) for AMPL. We do not compare the execution speed of the allocators, because they have been implemented in different languages.

\begin{tabular}{|l|cc|}
\hline RA & \multicolumn{2}{|c|}{ \#LOC } \\
\hline & RA & Interface \\
\hline Naive & $48(\mathrm{~J})$ & $773(\mathrm{~J})$ \\
\hline UBC & $2766(\mathrm{~J})$ & $773(\mathrm{~J})$ \\
\hline IRC & $3538(\mathrm{~J})$ & $773(\mathrm{~J})$ \\
\hline Chordal & $4134(\mathrm{~J})$ & $773(\mathrm{~J})$ \\
\hline LS & $385(\mathrm{~J})$ & $1100(\mathrm{~J})$ \\
\hline RA & $495(\mathrm{~A})$ & $298(\mathrm{~A})$ \\
\hline SARA & $731(\mathrm{~A})$ & $400(\mathrm{~A})$ \\
\hline
\end{tabular}

Fig. 7. Comparison between different register allocators plugged on RALF

We have plugged each of the eight algorithms into RALF and then tested the produced code on a StrongARM/XScale processor, with 64MB SDRAM, and no cache. We have drawn our benchmark programs from a variety of sources. We chose these benchmarks in part because the more traditional ones (for example, SPEC) have a huge memory print, and cannot be run in our resource constrained ARM hardware.

- Stanford Benchmark suite: a collection of seven programs that test recursive calls and array indexing.

- NetBench [19]: url is a network related benchmark that implements HTTP based switching; md5 is a typical cryptographic algorithm.

- Pointer-intensive benchmark [7: This benchmark suite is a collection of pointer-intensive benchmarks. Yacr2 is an implementation of a maze solver and $\mathrm{Ft}$ is an implementation of a minimum spanning tree algorithm. 


\begin{tabular}{|c|c|c|c|c|c|c|c|c|c|c|}
\hline & LOC & RTL & gcc-O2 & LS & $\mathrm{RA}$ & SARA & Chordal & $\overline{\mathrm{IRC}}$ & $\overline{\mathrm{UCB}}$ & Naive \\
\hline Bench & & & mem csr & mem csr & mem & em & em & em & nem & men \\
\hline Stanford & 307 & 1082 & 81 & $\begin{array}{lll}171 & 107\end{array}$ & 63 & $\overline{24}$ & $\begin{array}{|ll|}34 & 134\end{array}$ & $\begin{array}{lll}70 & 100\end{array}$ & 44133 & 854 \\
\hline yacr2 & 3979 & 10838 & \begin{tabular}{|l|}
1078289 \\
\end{tabular} & 3035335 & 1003123 & 1109142 & $\begin{array}{|ll|}2121 & 357\end{array}$ & 1957314 & 2200361 & 8181 \\
\hline $\mathrm{ft}$ & 2155 & 3218 & 299130 & 538151 & $\begin{array}{ll}225 & 87\end{array}$ & 230106 & 371162 & 561100 & 360169 & 2184 \\
\hline $\mathrm{c} 4$ & 897 & 40948 & $187 \quad 123$ & 715301 & 176145 & 179151 & 416170 & 453145 & 394170 & 3531 \\
\hline $\mathrm{mm}$ & 885 & 3388 & 386 & $\begin{array}{|ll|}2494 & 68 \\
\end{array}$ & 375116 & 380 & 591 & 648 & 687 & 2590 \\
\hline url & 652 & 1264 & 102 & 313 & $\begin{array}{ll}120 & 56\end{array}$ & 120 & 155 & 201 & 203 & 860 \\
\hline md5 & $\overline{790}$ & 3464 & 519110 & \begin{tabular}{|l}
1869433 \\
\end{tabular} & $\overline{500120}$ & 500120 & 570228 & $697 \quad 174$ & 580229 & 2714 \\
\hline
\end{tabular}

Fig. 8. Compile time statistics

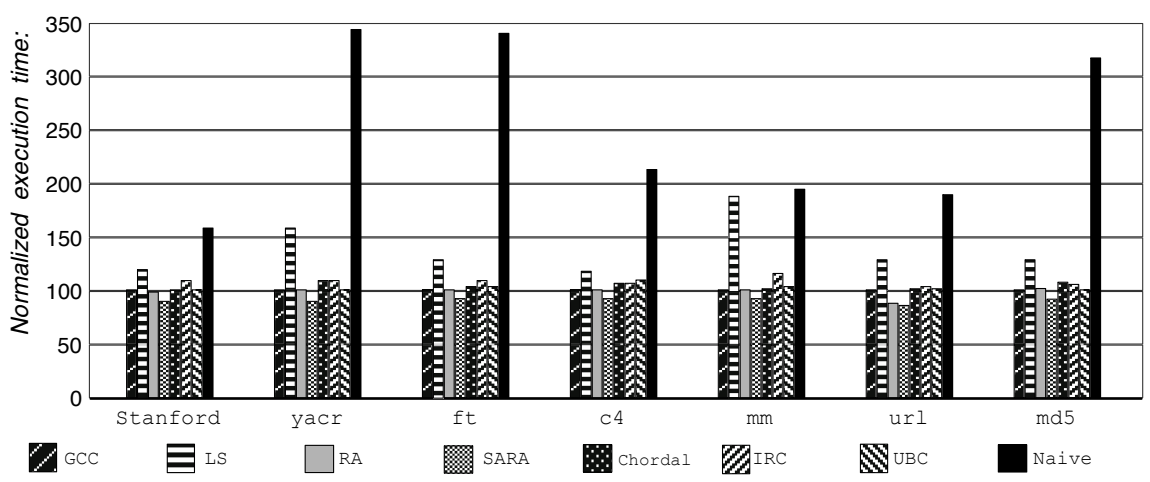

Fig. 9. Comparison of different register allocators using execution time of benchmarks as the metric

- c4 and mm are taken from the comp.benchmarks USENET newsgroup at http://www.cs.wisc.edu/ ${ }^{\sim}$ thomas/comp.benchmarks.FAQ.html. c4 is an implementation of the connect-4 game, and $\mathrm{mm}$ is a matrix multiplication benchmark.

The number of lines of $\mathrm{C}$ code (LOC) and the number of instructions in the RTL for these benchmarks are presented in Figure 8, These benchmarks are non-floating point programs. (We had to edit few of the programs to remove some code that uses floating point operations; we did so only after ensuring that the code with floating point operations is not critical to the behavior of the program.) For each benchmark, Figure 8 presents two static compile time statistics: the number of memory accesses (mem) due to spill/reload instructions, and the number of callee save registers (csr) used by the register allocator (leads to more memory accesses).

The chart in Figure 9 compares the execution times of the programs produced by each of the register allocators. The execution times have been normalized against the time obtained by programs compiled with gcc at the -O2 optimization level. It can be noted that, although the gcc algorithm is heavily tuned for the StrongARM architecture, Chordal, UBC, and IRC present comparative 
performances. Also Chordal and IRC's performances are similar, which confirms the results found by Pereira et al 27. Figure 9 suggests an upper limit on the gains that any register allocator can make. Even in the most extreme case, the code generated by the naive allocator is worse by a factor of 2.5 (as compared to the optimal solution found by the ILP based allocator). An important point is that most of these benchmarks deal with structures and arrays that require compulsory memory accesses, and it seems that these accesses overshadow the spill cost and hence such a small (2.5 times) improvement. Another conclusion is that the obtained execution time given by optimal solutions (SARA and RA), that run in worst-case exponential time, is not much lower than that obtained by polynomial time heuristics. It should be pointed that our experiments compare specific implementations of the allocators, and are run on a specific target machine; however, it was our objective to be as faithful as possible to the original description of each algorithm.

\section{Conclusion}

We have presented a framework that facilitates the development of register allocation algorithms. Our framework consists of the two description languages MIRA and FORD, plus a type system. Our framework is easy to use, and versatile enough to support a wide variety of register allocation paradigms. In order to validate this claim, we have developed RALF, an implementation of our framework for the StrongARM architecture, and used it to compare eight different register allocators.

Our framework has several limitations which may be overcome in future work: FORD does not permit the register allocator to modify the control flow graph of the target program; the grammar of MIRA supports only intra-procedural register allocation; the validation algorithm does not handle bitwidth aware register allocation 29]; and FORD does not support the concepts of re-materialization or code-motion. The implementation of RALF itself has the limitations that it targets only the ARM architecture, and RALF does not handle pseudos of type float or double (we have opted for this restriction to keep the implementation simple). So far we have experimented with only the C front-end of gcc; RALF can be seamlessly used with any language supported by gcc. Currently, we are extending RALF to allow the register allocator to do bitwidth-sensitiveanalysis.

RALF, our benchmarks, our Twelf proof, and a collection of tools that we have developed to aid in the design and test of register allocators are publicly available at http://compilers.cs.ucla.edu/ralf.

Acknowledgments. We thank the anonymous reviewers for comments on a draft of the paper. We were supported in part by a National Science Foundation Award number 0401691. Fernando Pereira was sponsored by the Brazilian Ministry of Education under grant number 218603-9. 


\section{References}

1. Standard ML of New Jersey (2000), http://www.smlnj.org/

2. GNU C compiler (2005), http://gcc.gnu.org

3. Adl-Tabatabai, A.-R., Gross, T., Lueh, G.-Y.: Code reuse in an optimizing compiler. In: OOPSLA, pp. 51-68. ACM Press, New York (1996)

4. Agat, J.: Types for register allocation. In: Clack, C., Hammond, K., Davie, T. (eds.) IFL 1997. LNCS, vol. 1467, pp. 92-111. Springer, Heidelberg (1998)

5. Andersson, C.: Register allocation by optimal graph coloring. In: Hedin, G. (ed.) CC 2003 and ETAPS 2003. LNCS, vol. 2622, pp. 34-45. Springer, Heidelberg (2003)

6. Appel, A.W, George, L.: Optimal spilling for CISC machines with few registers. In: PLDI, pp. 243-253. ACM Press, New York (2001)

7. Austin, T.M., Breach, S.E., Sohi, G.S.: Efficient detection of all pointer and array access errors. In: PLDI, pp. 290-301 (1994)

8. Chaitin, G.J.: Register allocation and spilling via graph coloring. SIGPLAN Notices 17(6), 98-105 (1982)

9. Elleithy, K.M., Abd-El-Fattah, E.G.: A genetic algorithm for register allocation. In: Ninth Great Lakes Symposium on VLSI, pp. 226-227 (1999)

10. Fourer, R., Gay, D.M., Kernighan, B.W.: AMPL A modeling language for mathematical programming. Scientific Press (1993), http://www.ampl.com

11. Freiburghouse, R.A.: Register allocation via usage counts. Commun. ACM 17(11), 638-642 (1974)

12. Fu, C., Wilken, K.: A faster optimal register allocator. In: MICRO, pp. 245-256. IEEE Computer Society Press, Los Alamitos (2002)

13. George, L., Appel, A.W.: Iterated register coalescing. TOPLAS 18(3), 300-324 (1996)

14. Goodwin, D.W., Wilken, K.D.: Optimal and near-optimal global register allocations using 0-1 integer programming. SPE 26(8), 929-968 (1996)

15. Hall, M.W., Anderson, J.-A.M., Amarasinghe, S.P., Murphy, B.R., Liao, S.-W., Bugnion, E., Lam, M.S.: Maximizing multiprocessor performance with the SUIF compiler. IEEE Computer 29(12), 84-89 (1996)

16. Huang, Y., Childers, B.R., Soffa, M.L.: Catching and identifying bugs in register allocation. In: Yi, K. (ed.) SAS 2006. LNCS, vol. 4134, Springer, Heidelberg (2006)

17. Leroy, X.: Formal certification of a compiler back-end or: programming a compiler with a proof assistant. In: POPL, pp. 42-54. ACM Press, New York (2006)

18. Lueh, G.-Y., Gross, T., Adl-Tabatabai, A.-R.: Global register allocation based on graph fusion. In: Languages and Compilers for Parallel Computing, pp. 246-265 (1996)

19. Memik, G., Mangione-Smith, B., Hu, W.: Netbench: A benchmarking suite for network processors. In: IEEE International Conference Computer-Aided Deisgn (November 2001)

20. Morrisett, G., Walker, D., Crary, K., Glew, N.: From system F to typed assembly language. TOPLAS 21(3), 527-568 (1999)

21. Naik, M., Palsberg, J.: Compiling with code size constraints. Transactions on Embedded Computing Systems 3(1), 163-181 (2004)

22. Krishna Nandivada, V., Palsberg, J.: Efficient spill code for SDRAM. In: CASES, pp. 24-31 (2003)

23. Krishna Nandivada, V., Palsberg, J.: Sara: Combining stack allocation and register allocation. In: Bodik, R. (ed.) CC 2005. LNCS, vol. 3443, pp. 232-246. Springer, Heidelberg (2005) 
24. Necula, G.C.: Translation validation for an optimizing compiler. In: PLDI, pp. 83-95. ACM Press, New York (2000)

25. Necula, G.C., Lee, P.: The design and implementation of a certifying compiler. In: PLDI, pp. 333-344 (1998)

26. Ohori, A.: Register allocation by proof transformation. Science of Computer Programming 50(1-3), 161-187 (2004)

27. Pereira, F.M.Q., Palsberg, J.: Register allocation via coloring of chordal graphs. In: ASPLAS (2005)

28. Poletto, M., Sarkar, V.: Linear scan register allocation. TOPLAS 21(5), 895-913 (1999)

29. Tallam, S., Gupta, R.: Bitwidth aware global register allocation. In: POPL, pp. 85-96 (2003)

30. Traub, O., Holloway, G.H., Smith, M.D.: Quality and speed in linear-scan register allocation. In: PLDI, pp. 142-151 (1998)

31. Vallee-Rai, R., Co, P., Gagnon, E., Hendren, L., Lam, P., Sundaresan, V.: Soot a Java bytecode optimization framework. In: CASCON (1999)

32. Pfenning, F., Schürmann, C.: System description: Twelf - a meta-logical framework for deductive systems. In: Ganzinger, H. (ed.) Automated Deduction - CADE-16. LNCS (LNAI), vol. 1632, pp. 202-206. Springer, Heidelberg (1999) 